\title{
Design and Implementation of Movie Recommendation System Based on Knn Collaborative Filtering Algorithm
}

\author{
Bei-Bei CUI \\ School of Software Engineering, Beijing University of Technology, Beijing, China \\ e-mail: cuibeibeibatty@163.com
}

\begin{abstract}
In the spread of information, how to quickly find one's favorite movie in a large number of movies become a very important issue. Personalized recommendation system can play an important role especially when the user has no clear target movie. In this paper, we design and implement a movie recommendation system prototype combined with the actual needs of movie recommendation through researching of KNN algorithm and collaborative filtering algorithm. Then we give a detailed principle and architecture of JAVAEE system relational database model. Finally, the test results showed that the system has a good recommendation effect.
\end{abstract}

\section{Introduction}

With the rapid development of Internet technology[1], today's society has entered the era of Web 2, information overload has become a reality. How to find the required information in the mass of data has become a hot research topic. Movie is one of the main spiritual entertainment, also has the problem of information overload. In order to solve this problem, this paper put forward a proposal of personalized movie recommendation system[1,2].

Personalized recommendation try to know the characteristics and preferences of the user by collecting and analysing historical behavior to know what kind of person the user is, what kind of behavior preference the user has, what kind of things the user like to share and so on[3.4.5], and finally understand that user characteristics and preferences based on the rules of the platform and recommend the information and goods which the user interested[6.7]. Personalized recommendation system is a kind of information filtering technology. It is an integrated system which is a combination of a variety of data mining algorithms and user related information, to meet the interests or potential interests of users. The common recommendation system is categorized as content based recommendation system, collaborative filtering recommendation system, and hybrid recommendation system[9,10]. Each recommendation algorithm has different use range and use condition, it results in the use of different recommendation algorithm for the same information recommendation. In the actual application of recommendation system, the system tends to be a hybrid recommendation system. That is, to mix the advantage of each recommendation algorithm to the recommended process to effectively improve the recommendation effect.

In this paper, the key research contents is to help users to obtain user-interested movie automatically in the massive movie information data using KNN algorithm and collaborative filtering algorithm, and to develop a prototype of movie recommendation system based on KNN collaborative filtering algorithm.

\section{Literature Survey}

\subsection{KNN algorithm}

KNN algorithm is called $\mathrm{K}$ nearest neighbor classification algorithm. The core idea of the KNN algorithm is: if the majority of the $\mathrm{k}$ most similar neighbors of sample in the feature space belongs to a certain category, then the sample is considered to belong to this category[8]. As shown in Figure 1 , the majority of w's nearest neighbors belong to the $\mathrm{x}$ category, w belongs to the $\mathrm{X}$ category. 


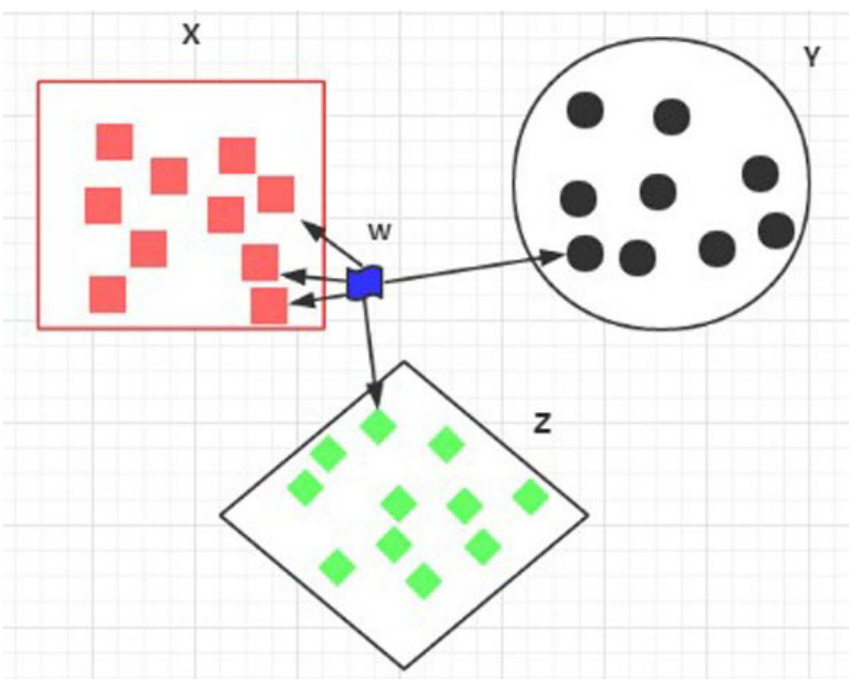

Fig. 1. example of KNN algorithm

\subsection{Collaborative filtering algorithm}

Collaborative filtering algorithm is categorized as user-based collaborative filtering algorithm[4] and project-based collaborative filtering. The basic principles of the two is quite similar, and this section mainly introduces the user-based collaborative filtering recommendation algorithm. The basic idea of collaborative filtering recommendation algorithm is to introduce the information of similar-interest users to object users[7]. As shown in figure 2.
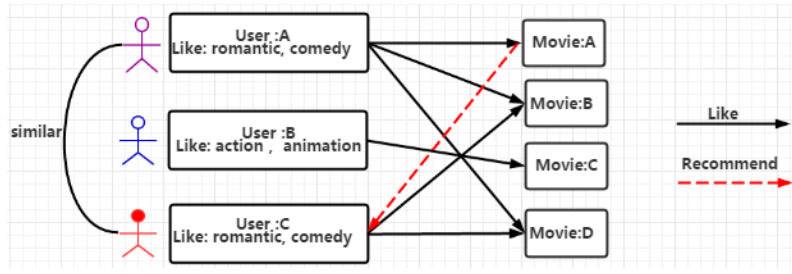

Fig. 2. example of UserCF algorithm

User A loves movie A, B, C, and user C likes movie B, D, so we can conclude that the preferences of user $A$ and user $C$ are very similar. Since user A loves movie D as well, so we can infer that the user A may also love item D, therefore item $\mathrm{D}$ would be recommended to the user. The basic idea of the algorithm is based on records of history score of user. Find the neighbor user as $u$ ' who has the similar interest with target user $\mathrm{u}$, and then recommend the items which the neighbor user $u^{\prime}$ loved to target user $\mathrm{u}$, the predict score which target user $u$ may give on the item is obtained by the score calculation of neighbor user $u^{\prime}$ on the item. The algorithm consists of three basic steps: user similarity calculation, nearest neighbor selection and prediction score calculation.

\section{KNN collaborative filtering algorithm}

KNN collaborative filtering algorithm, which is a collaborative filtering algorithm combined with KNN algorithm, use KNN algorithm to select neighbors. The basic steps of the algorithm are user similarity calculation, KNN nearest neighbor selection and predict score calculation[11.12].

\subsection{User similarity computing}

The similarity between users is calculated by evaluating the value of the items evaluated by two users.

Each user uses $\mathrm{N}$ dimension vector to represent item score, for example, to calculate of similarity of U1 and U3, first find out the set of films that they all scored as $\{\mathrm{m} 1, \mathrm{M} 2, \mathrm{M} 4, \mathrm{~m} 5\}$ and relative scores of these films. The score vector of U1 is $\{1,3,4,2\}$, and the score vector of U3 is $\{2,4,1,5\}$. The similarity of U1 and U3 is calculated by the similarity formula[13].

\begin{tabular}{|l|l|l|l|l|l|}
\hline U\M & $\mathrm{m} 1$ & $\mathrm{~m} 2$ & $\mathrm{~m} 3$ & $\mathrm{~m} 4$ & $\mathrm{~m} 5$ \\
\hline $\mathrm{u} 1$ & 1 & 3 & 3 & 4 & 2 \\
\hline $\mathrm{u} 2$ & 3 & 1 & 4 & & \\
\hline u3 & 2 & 4 & & 1 & 5 \\
\hline $\mathrm{u} 4$ & 2 & & 2 & & \\
\hline
\end{tabular}

Fig. 3. Calculating formula of users' similarity

The similarity of $\mathrm{u}$ and $u^{\prime}$ is denoted as $\operatorname{sim}\left(u, u^{\prime}\right)$, the commonly used method of calculating user similarity are Cosine Similarity and Pearson Correlation similarity.

\subsubsection{Cosine similarity}

The method calculates the similarity between two users by calculating the cosine of the angle between the two vectors ${ }^{[2]}$ :

$\operatorname{sim}\left(x, y^{\prime}\right)=\cos (\vec{X}, \vec{Y})=\frac{\vec{X} * \vec{Y}}{|\vec{X}| *|\vec{Y}|}=\frac{\sum_{s \in s_{x y}} r_{x, s} r_{y, s}}{\sqrt{\sum_{s \in s_{x y}}\left(r_{x, s}\right)^{2}} \sqrt{\sum_{s \in s_{x y}\left(r_{y, s}\right)^{2}}}}$

Among them, $r_{x, s}$ and $r_{y, s}$ are the score of goods s scored by user $\mathrm{X}$ and $\mathrm{Y}$ respectively. $s_{x y}$ is the set of movies that user $\mathrm{x}$ and user $\mathrm{y}$ both scored on. In other words, $s_{x y}=\left\{s \in \text { Items } \mid r_{x, s} \neq \varepsilon \cap r_{y, s} \neq \varepsilon\right\}^{[3]}$

\subsubsection{Pearson correlation similarity}

Pearson correlation similarity is a measurement of the linear relationship between two variables. 


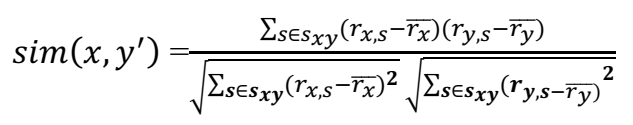

Among them, $\overline{r_{x}}$ is the average score is $\mathrm{x}[3]$, the rest of the symbolic meaning is the same as formula(1).

\subsection{KNN nearest neighbor selection}

After the calculation of similarity as $\operatorname{sim}\left(u, u^{\prime}\right)$ between users, then the algorithm selects a number of users the highest similarity as the U's neighbor, denoted as u'. set a fixed value $\mathrm{K}$ for the neighbor selection, select only the most $\mathrm{K}$ high similarity as neighbors regardless of the value of the neighbor similarity of users. As shown in figure 4.

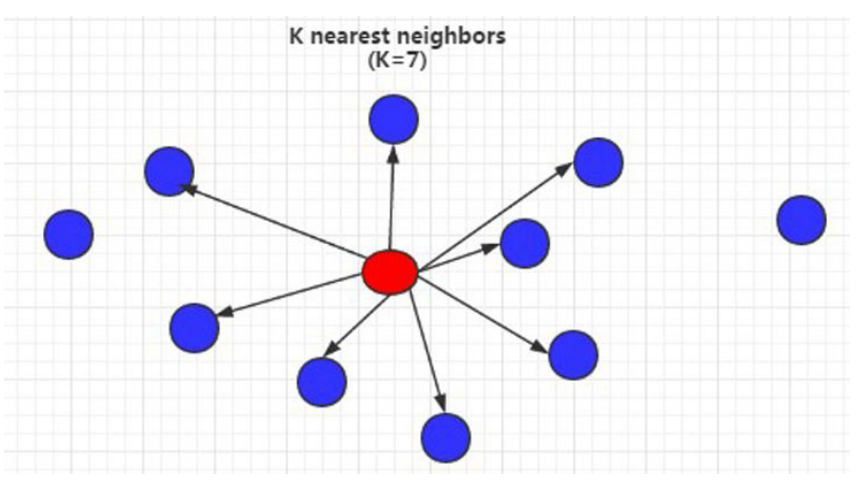

Fig.4. formula of $\mathrm{K}$ nearest neighbors when $\mathrm{k}=7$

\subsection{Predict score calculation}

After determining the user's neighbors, the score can be predicted according to the score of the neighbor to the item, The calculation formula is as follows:

$r_{u, \mathrm{i}}=\bar{r}_{u}+\mathrm{k} \sum_{u \prime \in U} \operatorname{sim}\left(u, u^{\prime}\right) *\left(r_{u^{\prime}, \mathrm{i}}-\bar{r}_{u^{\prime}}\right)$

$\left(\mathrm{k}=1 / \sum\left|\operatorname{sim}\left(u, u^{\prime}\right)\right|\right)$

$r_{u, \mathrm{i}}$ was used to predict the score of user $\mathrm{u}$ to movie i[6].

To sum up, the process of calculating prediction score of user $\mathrm{u}$ for $\mathrm{i}$ is as follows:

Step1. Generate user - item two-dimensional matrix of score as Rmxn, where each score is $r_{u, \mathrm{i}}$.

Step2: Use principle of cosine similarity or Pearson correlation similarity to calculate the similarity between each 2 users as $\operatorname{sim}\left(u, u^{\prime}\right)$, and generate the user similarity matrix.

Step3: according to the results obtained by Step2, find K number of score which has the maximum weight, the corresponding $\mathrm{K}$ users is the neighbors of $\mathrm{u}$.

Step4: Use formula 3 to calculate the predictive value of $\mathrm{i}$ for target user $\mathrm{u}$.

In this way, we can calculate the prediction score of the target users for the non-scored movies, and the $\mathrm{N}$ movies with the highest score can be recommended to the user.

In this paper, KNN collaborative filtering algorithm based on user is used to implement the recommendation of movie[], and the collaborative filtering algorithm based on the project is used to implement the recommendation of the associated movie[5]. In addition, it can also recommend the movies to new users according to user registration information, and it can make new and unpopular movie recommendation according to the film's browsing and score[14].

\section{Personalized system design}

recommendation

\section{1 architecture design}

The system is based on $\mathrm{B} / \mathrm{S}$ mode, uses JavaEE architecture, Tomcat server for system deployment, the architecture is shown in figure 5.

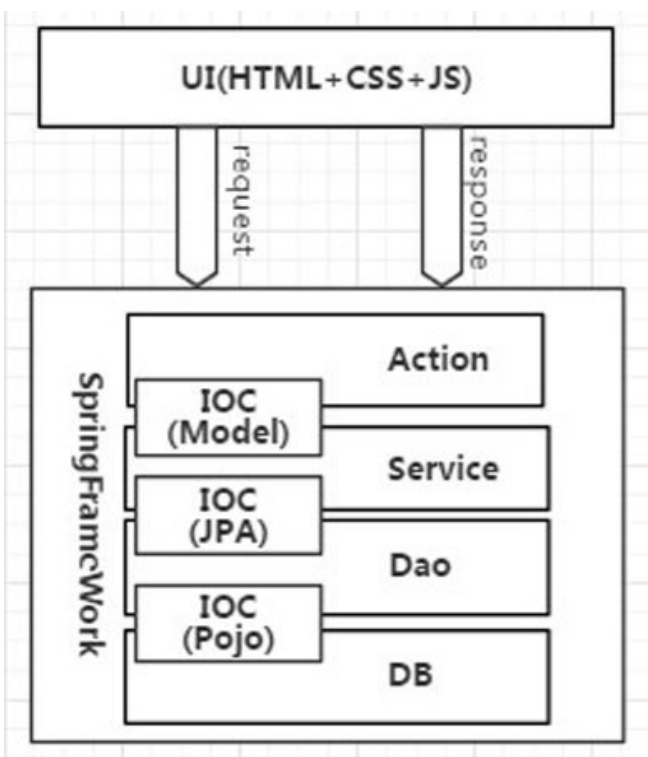

Fig. 5. System Architecture

Front view is implemented using HTML, CSS, JAVASCRIPT, the back end uses Struts2, Spring and Hibernate, the database uses MySQL for storage. The system is object-oriented to guarantee system of high cohesion and improve development efficiency using the SSH protocol[17]. Besides, it enhances the maintainability and scalability by separating Controller layer and View layer to reduce the degree of coupling between them, making it easier to maintain and modify the WEB application.

\subsection{Database design}

Database is the basis of the system, this system uses MYSQL database, the overall database structure diagram is shown in the following figure 6 , representing the integrity constraints between the data tables[18]. 


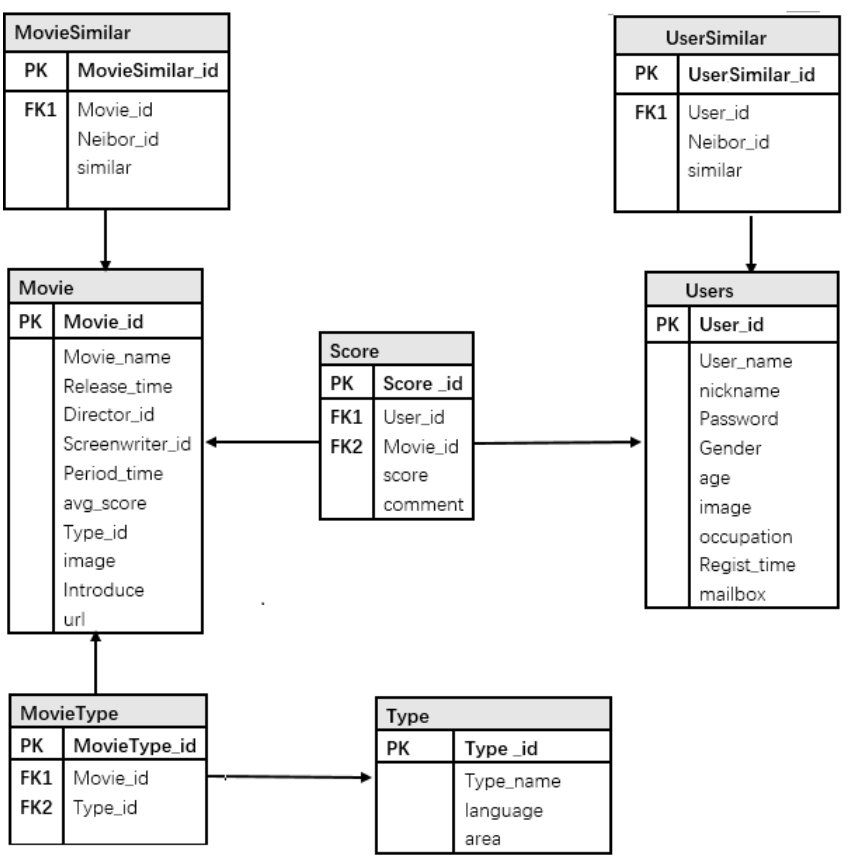

Fig. 6. Relational Model of Database

Table Users is the description of user information, including user ID, user name, password, registration time, etc. Table UserSimilar is the description of the user similarity information, including the user similarity ID, user ID, similar neighbor user ID, and the value of the similarity. Table Score is the description of users' rating information on the film, which is the direct information source of collaborative filtering algorithm, it includes the score ID, the user's ID who give the score ,the value of the score, content of comments. Table Movie is the description of the movie information, including the movie ID, movie name, director, movie URL, etc. Table MovieType is the description of type information of the movies, including the ID of movies' type, movie name, and type ID. Table MovieSimilar is the description of the movie similarity information, including the movie similarity ID, movie ID, the ID of highly similar neighbor, the value of similarity. Both the table UserSimilar and table Moviesimilar are the basis of the recommendation algorithm and system $^{[15.16]}$.

\section{System operation effect}

user registration system will capture the user's explicit and implicit behavioral characteristics and these characteristics is stored in the user database through the user login module. After logging in the system, the system will make the appropriate recommendation according to the user's information[19.20]. As shown in figure 7.

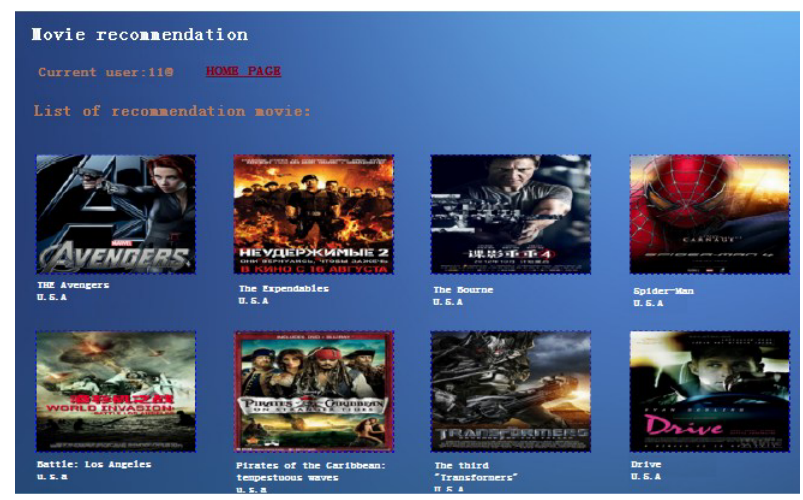

Fig. 7. the display of personal recommendation list

\section{Conclusion}

Under the condition of massive information, the requirements of movie recommendation system from film amateur are increasing. This article designs and implements a complete movie recommendation system prototype based on the KNN algorithm, collaborative filtering algorithm and recommendation system technology[10]. We give a detailed design and development process, and test the stability and high efficiency of experiment system through professional test. This paper has reference significance for the development of personalized recommendation technology.

\section{References}

[1] Peng, Xiao, Shao Liangshan, and Li Xiuran. "Improved Collaborative Filtering Algorithm in the Research and Application of Personalized Movie Recommendations", 2013 Fourth International Conference on Intelligent Systems Design and Engineering Applications, 2013.

[2] Munoz-Organero, Mario, Gustavo A. Ramíez-González, Pedro J. Munoz-Merino, and Carlos Delgado Kloos. "A Collaborative Recommender System Based on SpaceTime Similarities", IEEE Pervasive Computing, 2010.

[3] Al-Shamri, M.Y.H.. "Fuzzy-genetic approach to recommender systems based on a novel hybrid user model", Expert Systems With Applications, 200810

[4] Hu Jinming. "Application and research of collaborative filtering in e-commerce recommendation system", 2010 3rd International Conference on Computer Science and Information Technology, 07/2010

[5] Xu, Qingzhen Wu, Jiayong Chen, Qiang. "A novel mobile personalized recommended method based on money flow model for stock exchange.(Researc", Mathematical Problems in Engineering, Annual 2014 Issue

[6] Yan, Bo, and Guanling Chen. "AppJoy : personalized mobile application discovery", Proceedings of the 9th international conference on Mobile systems applications and services - MobiSys 11 MobiSys 11, 2011.

[7] Davidsson C, Moritz S. Utilizing implicit feedback and context to recommend mobile applications from first use. 
In: Proc. of the Ca RR 2011. New York: ACM Press, 2011.

22.http://dl.acm.org/citation.cfm?id=1961639[doi:10.11 45/1961634.1961639]

[8] Bilge, A., Kaleli, C., Yakut, I., Gunes, I., Polat, H.: A survey of privacy-preserving collaborative filtering schemes. Int. J. Softw. Eng. Knowl. Eng. 23(08), 10851108 (2013)CrossRefGoogle Scholar

[9] Calandrino, J.A., Kilzer, A., Narayanan, A., Felten, E.W., Shmatikov, V.: You might also like: privacy risks of collaborative filtering. In: Proceedings of the IEEE Symposium on Security and Privacy, pp. 231-246, Oakland, CA, USA (2011)

[10] Research.ijcaonline.org

[11] Hu Jinming. "Application and research of collaborative filtering in e-commerce recommendation system", 2010 3rd International Conference on Computer Science and Information Technology, 07/2010

[12] www.pacis2014.org

[13] Xie HT, Meng XW. A personalized information service model adapting to user requirement evolution. Acta Electronica Sinica, 2011,39(3):643-648 (in Chinese with English abstract).

[14] Polat, H., Du, W.: Privacy-preserving top-n recommendation on distributed data. J. Am. Soc. Inf. Sci. Technol. 59(7), 1093-1108 (2008). http://dx.org/10.1002/asi.20831CrossRefGoogle Scholar
[15] Su, X., Khoshgoftaar, T.M.: A survey of collaborative filtering techniques. Adv. Artif. Intell, p. 4 (2009). http://dx.org/10.1155/2009/421425

[16] Yakut, I., Polat, H.: Estimating NBC-based recommendations on arbitrarily partitioned data with privacy. Knowl. Based Syst. 36(0), 353362(2012). http://www.sciencedirect.com/science/ar ticle/pii/S0950705112002031CrossRefGoogle Scholar

[17] Okkalioglu, M., Koc, M., Polat, H.: On the discovery of fake binary ratings. In: Proceedings of the 30th Annual ACM Symposium on Applied Computing, SAC 2015, pp. 901-907. ACM, USA (2015). http://doi.acm.org/10.1145/2695664.2695866

[18] Wang LC, Meng XW, Zhang YJ. A cognitive psychology-based approach to user preferences elicitation for mobile network services. Acta Electronica Sinica, 2011,39(11):2547-2553 (in Chinese with English abstract)

[19] Kaleli, C., Polat, H.: Privacy-preserving naïve bayesian classifier based recommendations on distributed data. Comput. Intell. 31(1), 4768(2015). http://dx.org/10.1111/coin.12012MathSciNet CrossRefGoogle Scholar

[20] Munoz-Organero, Mario, Gustavo A. Ramíez-González, Pedro J. Munoz-Merino, and Carlos Delgado Kloos. "A Collaborative Recommender System Based on SpaceTime Similarities", IEEE Pervasive Computing, 2010. 